\title{
Secondary brain abscess following simple renal cyst infection: a case report
}

\author{
Nobuhiro Akuzawa ${ }^{1 *}$, Tenshi Osawa ${ }^{2}$, Masayuki Totsuka ${ }^{1}$,Takashi Hatori ${ }^{1}$, Kunihiko Imai ${ }^{1}$, Yonosuke Kitahara ${ }^{1}$ \\ and Masahiko Kurabayashi ${ }^{3}$
}

\begin{abstract}
Background: Escherichia coli (E. coli) is the most common causative bacteria of neonatal meningitis, but hematogenous intracranial $E$. coli infection is rare in adults. Moreover, intracranial abscess formation owing to E. coli, including brain abscesses and subdural empyema formation, is extremely rare. We herein present a case involving a patient with a brain abscess owing to E. coli following a simple renal cyst infection. A review of the literature is also presented.

Case presentation: A 77-year-old Japanese woman with a history of polymyalgia rheumatica was admitted to our hospital because of persistent fever, right flank pain, and pyuria. Intravenous antibiotics were administered; however, her level of consciousness deteriorated 6 days after admission. Contrast-enhanced magnetic resonance imaging showed a brain abscess in the left occipital lobe and pyogenic ventriculitis. Enhanced abdominal computed tomography revealed a right renal cyst with heterogeneous content. Culture of urine, blood, and aspirated pus from the infected cyst revealed E. coli with identical antibiotic sensitivity in all sites, suggesting that the cyst infection and subsequent bacteremia might have caused the brain abscess. The patient recovered after a 6-week course of meropenem.
\end{abstract}

Conclusion: The prognosis of patients with E. coli-associated intracranial abscess is usually poor. Advanced age and immunosuppression may be potent risk factors for intracranial abscess formation owing to the hematogenous spread of E. coli.

Keywords: Bacteremia, Brain abscess, Escherichia coli, Simple renal cyst

\section{Background}

Although brain abscesses are relatively uncommon, they remain potentially fatal central nervous system (CNS) infections despite the evolution of neurosurgical techniques, new antibiotics, and new imaging technologies. Brain abscesses are usually caused by contiguous infections such as sinusitis or middle ear infections; however, hematogenous spread of pyogenic pathogens from remote-organ infections can also cause brain abscesses [1].

The clinical signs of brain abscesses are nonspecific. The most common symptoms are reportedly headache and fever, but the classical triad of fever, headache, and nausea is seen in up to $20 \%$ of patients [2]. Focal neurological deficits are reportedly recognized in $57 \%$ of

\footnotetext{
* Correspondence: akuzawa_nobuhiro@yahoo.co.jp

'Departments of Internal Medicine, 1-7-13 Koun-cho, Maebashi, Gunma 371-0025, Japan

Full list of author information is available at the end of the article
}

patients [2]. Additionally, laboratory findings may show a normal white blood cell (WBC) count or C-reactive protein (CRP) level [2]. Thus, early imaging studies including enhanced brain computed tomography $(\mathrm{CT})$ or magnetic resonance imaging (MRI) are of cardinal importance for a definitive diagnosis and appropriate treatment in the early period $[1,2]$.

We herein present a rare adult case of a left occipital lobe brain abscess that likely resulted from the hematogenous spread of an Escherichia coli (E. coli) right renal cyst infection followed by formation of pyogenic intraventricular empyema.

\section{Case presentation}

A 77-year-old Japanese woman was admitted to our hospital because of fever of unknown origin. She had developed general fatigue and a slight fever 2 weeks before admission. Headache and a fever higher than $38^{\circ} \mathrm{C}$ had developed 3 days before admission, and oral cefditoren 
pivoxil prescribed at another hospital had been ineffective. She had a 2-year history of polymyalgia rheumatica and chronic gastritis. She had initially been treated with $15 \mathrm{mg} /$ day of prednisolone for the polymyalgia rheumatica. Her dosage of prednisolone was tapered with improvement of her symptoms, and she had been treated with prednisolone $(5 \mathrm{mg} /$ day $)$ and famotidine (20 mg/day) for 1 year before admission. She had no other medical or family history and did not smoke or drink alcohol. Physical examination on admission revealed a height of $156 \mathrm{~cm}$, weight of $49 \mathrm{~kg}$, temperature of $40.2^{\circ} \mathrm{C}$, heart rate of 120 beats/min, and blood pressure of 116/ $62 \mathrm{mmHg}$. No obvious abnormalities of the chest or abdomen were found with the exception of slight right flank pain. Neurological examination also showed normal findings; her Glasgow Coma Scale (GCS) score was maximal at 15 (E4V5M6). She was ambulatory; no symptoms suggesting agnosia, agraphia, or any other higher brain dysfunction were observed on admission. In addition, finger perimetry showed no obvious visual field deficits. Laboratory findings showed a high WBC count $\left(20,200 / \mathrm{mm}^{3}\right)$, platelet count $\left(40.4 \times 10^{4} / \mathrm{mm}^{3}\right)$, and CRP level $(10.02 \mathrm{mg} / \mathrm{dl})$. Chest X-ray, abdominal $\mathrm{X}$-ray, and electrocardiographic findings were normal. Urinalysis revealed pyuria with markedly increased WBCs and gram-negative bacilli, suggesting a urinary tract infection. Intravenous administration of ampicillin (6 g/day) was begun immediately after admission. On day 4 , her body temperature remained higher than $38^{\circ} \mathrm{C}$ despite improvement in the WBC count $\left(18,300 / \mathrm{mm}^{3}\right)$ and CRP level $(3.91 \mathrm{mg} / \mathrm{dl})$. On the same day, E. coli was revealed in urine and blood culture specimens taken on admission. Although the $E$. coli was sensitive to ampicillin, we substituted ceftriaxone ( $2 \mathrm{~g} /$ day) for ampicillin on day 4 based on the results of the antibiotic sensitivity test.

On day 5 , her body temperature dropped to $37.5^{\circ} \mathrm{C}$. However, she began to drop her spoon frequently during meals and complain of difficulty in donning her shirt. On day 6 , she complained of difficulty in seeing. Although she did not exhibit severe palsy or ataxia, she could no longer eat a meal, change her clothes, or walk by herself without nursing care. She was considerably confused, and it was difficult to evaluate her neurological function precisely. Her level of consciousness sharply deteriorated thereafter, and her GCS score dropped to 9 (E2V3M4). Her WBC count and CRP level on day 6 were $15,600 / \mathrm{mm}^{3}$ and $3.63 \mathrm{mg} / \mathrm{dl}$, respectively. Electrolyte, thyroid hormone, and blood glucose levels were normal. Plain brain CT on day 6 showed debris in the right lateral ventricle and a tumor-like lesion in the left posterior lobe (Figure 1A). Contrast-enhanced MRI on day 6 revealed a tumor-like lesion in the left posterior lobe with ring-like enhancement on T1-weighted imaging (Figure 1B). On diffusion-weighted imaging, this lesion also showed homogenous high intensity, which seemed to be connected with the left lateral ventricle, and obvious debris was present in both lateral ventriculi (Figure 1C). Based on these findings, we considered that ventricular rupture of a brain abscess was the most probable cause of her deterioration. The size of the abscess was $1.6 \times 1.8 \mathrm{~cm}$. Subsequent abdominal contrastenhanced CT on day 6 showed a unilocular right renal cyst with heterogeneous and high-density content, suggesting a renal cyst infection (Figure 2A). Abdominal ultrasonographic findings were inconclusive for a cyst infection. Contrast-enhanced chest CT showed no remarkable findings. Further laboratory and transthoracic echocardiographic diagnostics excluded potential risk factors for brain abscess, such as human immunodeficiency virus infection, fungal infection, endocarditis, and pulmonary hypertension. We immediately consulted with neurosurgeons and decided to manage the right occipital lesion with intravenous meropenem administration (6 g/day). Her consciousness gradually improved thereafter; her GCS score recovered to 12 (E3V4M5) on day 9. She was slightly confused and showed impaired orientation, but her ability to converse with us had improved. Brain MRI on day 9 revealed no remarkable changes in the size of the abscess. No signs of high intracranial pressure or hydrocephalus were present either clinically or radiographically. Cerebrospinal fluid (CSF) analysis on day 9 revealed a slight increase in leukocytes $\left(64 / \mathrm{mm}^{3}\right)$, including $23 / \mathrm{mm}^{3}$ (36\%) lymphocytes and $41 / \mathrm{mm}^{3}(64 \%)$ neutrophils with no atypical cells, as well as high total protein $(517 \mathrm{mg} / \mathrm{dl})$ and low glucose $(21 \mathrm{mg} / \mathrm{dl})$ levels. Microscopy of CSF smear samples with methylene blue staining, India ink capsule staining, and Gram's staining detected no bacteria or fungi. The CSF culture was sterile. On day 12, her body temperature remained below $37.5^{\circ} \mathrm{C}$, but her WBC count $\left(14,900 / \mathrm{mm}^{3}\right)$ and CRP level $(4.01 \mathrm{mg} / \mathrm{dl})$ remained high. Additionally, her right flank pain persisted despite antibiotic therapy. MRI on day 12 showed no deterioration of the brain abscess. Based on these findings, we concluded that CT-guided drainage was needed to manage the renal cyst infection. The drainage procedure was performed on day 13, and E. coli from the aspirated pus showed the same sensitivity to antibiotics as did the E. coli from the blood and urine on admission. The patient showed good progress thereafter. The drainage catheter was removed on day 20. Her GCS score recovered to 15 on day 22 . On day 30 , she showed almost normal activities of daily life, although she still experienced difficulty in seeing. Her WBC count and CRP level normalized, and enhanced brain MRI showed a dramatic decrease in the abscess size (Figure 1D). Moreover, abdominal enhanced CT on day 35 showed that the right renal cyst had decreased in size (Figure 2B). 


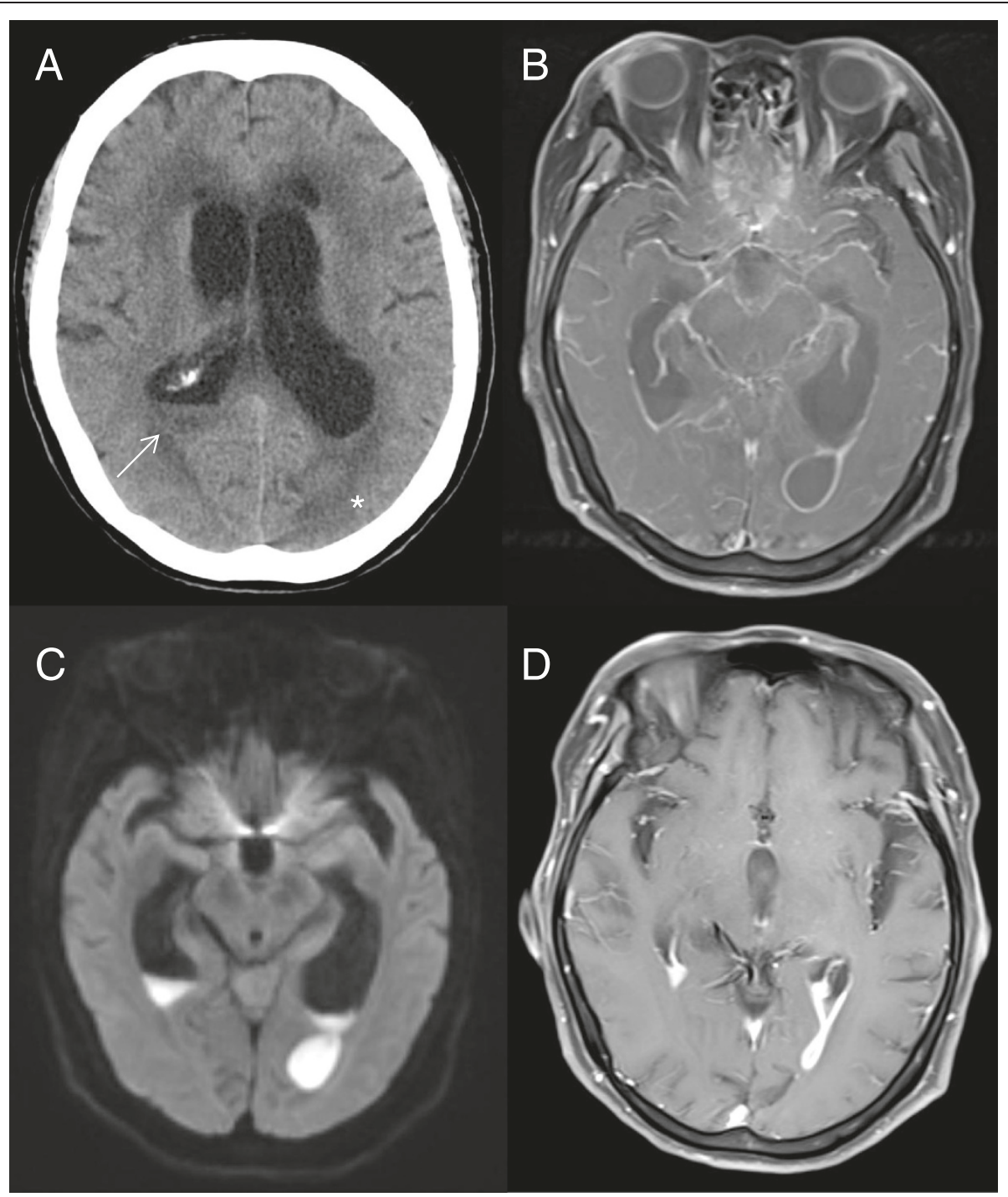

Figure 1 Plain computed tomography (CT) and contrast-enhanced magnetic resonance imaging (MRI) of the brain on days 6 and 30 . A: Plain $C T$ on day 6 . Debris in the right lateral ventricle (white arrow) and a space-occupying lesion with surrounding edema in the left occipital lobe (asterisk) were observed. B: T1-weighted, contrast-enhanced MRI on day 6. The left occipital lesion displayed ring-like enhancement. C: Diffusion-weighted imaging on day 6 . Homogenous high-intensity signaling of the lesion was observed. D: Contrast-enhanced T1-weighted imaging on day 30 . The left occipital lesion dramatically decreased in size.

Formal perimetry was performed by an ophthalmologist on day 35 and showed obvious right inferior quadrantic hemianopsia. Moreover, transesophageal echocardiography using contrast medium on day 35 showed no cryptic abnormalities, including intracardiac right-toleft shunting or small vegetations. Intravenous administration of meropenem was terminated on day 42 , and no deterioration was observed even in the absence of oral antibiotics. Following rehabilitation, she was discharged from the hospital on day 60 with right inferior quadrantic hemianopsia as an aftereffect. She has been followed up for 6 months since discharge, and no recurrence of the brain abscess or renal cyst infection has been observed.

\section{Discussion}

The development of a brain abscess owing to hematogenous spread of pathogens from a distant infectious focus, such as a lung abscess, empyema, skin infection, or intra-abdominal infection, is seen in $15-30 \%$ of cases [1]. In patients with a cryptogenic brain abscess, examination for potential cardiovascular diseases may reveal congenital heart diseases, patent foramen ovale, or arteriovenous fistula that can permit pathogenic bacteria to bypass the lungs and enter the systemic circulation [1]. Urinary tract infections may cause metastatic brain abscesses, and Enterobacteriaceae and Pseudomonas species have been reported as causative pathogens [1,2]. In general, however, aerobic and anaerobic streptococci 


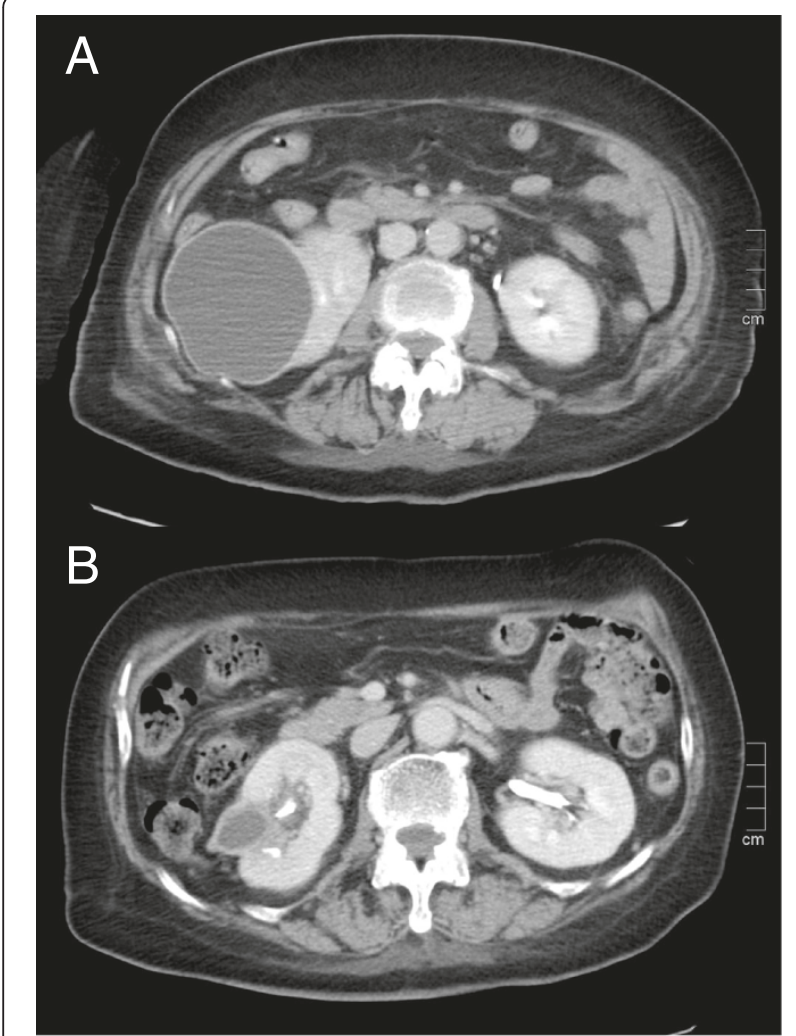

Figure 2 Contrast-enhanced computed tomography (CT) images of the abdomen on days 6 and 35. A: CT image on day 6 showed a right renal cyst $(9.3 \times 7.2 \mathrm{~cm})$. The $\mathrm{CT}$ attenuation value of the inner cyst contents was about 25 Hounsfield units, much higher than the value of water. The lateral margin of the cyst was faintly contrasted. B: CT image on day 35 . The right renal cyst had markedly decreased in size after the drainage procedure. are the most common pathogens in brain abscesses owing to contiguous and hematogenous spread of infection; the frequency of gram-negative bacterial infection is low $[1,2]$. Basically, E. coli and Group B Streptococcus are known to be common causative bacteria of neonatal meningitis [3]. Moreover, to the best of our knowledge, only nine adults with $E$. coli intracranial abscesses, including brain abscesses or subdural empyema, have been reported in the past 20 years (Table 1) [4-12]. Additionally, only two cases of $E$. coli brain abscess have been reported $[6,9]$. Interestingly, eight of the nine reported cases involved older men, implying that age and sex may be potent risk factors. Six patients died within 1 month after diagnosis [4-6,8,10,12]. In five cases, extracranial infection had been recognized prior to the onset, suggesting that the hematogenous spread of $E$. coli might be a pivotal cause of intracranial abscess formation $[4,5,7,9,11]$. Preceding urinary tract infection was reported in three cases $[4,7,11]$, and only one patient had a history of taking immunosuppressants (corticosteroids) [6]. These reported patients include one with a history of splenectomy [8], one with esophageal cancer undergoing radiochemotherapy [11], and two with diabetes $[6,10]$. These findings suggest that in addition to sex and age, immunosuppression may be another risk factor for intracranial $E$. coli infection. In our case, the E. coli strains obtained from urine, blood, and aspirated pus from the infected renal cyst showed the same minimum inhibitory concentration, suggesting a strong involvement of hematogenous infection. However, one limitation of our report was the inability to determine the genetic identity of these $E$. coli strains by analysis of restriction products after digestion of chromosomal deoxyribonucleic acid with restriction endonuclease.

Table 1 Reported adult cases of brain abscess or subdural empyema owing to Escherichia coli infection

\begin{tabular}{|c|c|c|c|c|c|c|c|c|}
\hline Author & Year & $\begin{array}{l}\text { Age } \\
\text { sex }\end{array}$ & $\begin{array}{c}\text { Immuno- } \\
\text { suppressants }\end{array}$ & Underlying diseases & $\begin{array}{l}\text { Preceding infection } \\
\text { opportunity }\end{array}$ & Lesion & $\begin{array}{l}\text { Surgical } \\
\text { treatment }\end{array}$ & Outcome \\
\hline Bakker [4] & 1995 & $88 \mathrm{~W}$ & $(-)$ & Column fracture & $\begin{array}{c}\text { Orthopedic surgery } \\
\text { (hip), UTI }\end{array}$ & SE & Drainage & Dead \\
\hline Hirano [5] & 1995 & $86 M$ & $(-)$ & Chronic cholecystitis & Cholecystitis & SE & Drainage & Dead \\
\hline Rickert [6] & 2000 & $52 M$ & Cortico- steroids & DCM, DM & $(-)$ & BA, Malaloplakia & Craniotomy & Dead \\
\hline Nishi [7] & 2005 & $76 \mathrm{M}$ & $(-)$ & ADPKD & Renal cyst infection & SE & Drainage & Alive \\
\hline Bachmeyer [8] & 2005 & $55 \mathrm{M}$ & $(-)$ & Esophageal cancer & $(-)$ & SE & $(-)$ & Dead \\
\hline Doepp [9] & 2006 & $67 M$ & $(-)$ & PFO & Perianal abscess & BA & $(-)$ & Alive \\
\hline Adamides [10] & 2007 & $91 \mathrm{M}$ & $(-)$ & $\begin{array}{l}\text { Chronic subdural } \\
\text { hematoma, DM }\end{array}$ & $\begin{array}{l}\text { Surgical aspiration of } \\
\text { subdural hematoma }\end{array}$ & SE & Drainage & Dead \\
\hline Narita [11] & 2009 & $80 \mathrm{M}$ & $(-)$ & $\begin{array}{l}\text { Post-gastrectomy } \\
\text { and splenectomy }\end{array}$ & $\begin{array}{l}\text { Orthopedic surgery } \\
\text { (leg, spine), UTI }\end{array}$ & SE & Drainage & Alive \\
\hline Redhu [12] & 2011 & $48 M$ & $(-)$ & $(-)$ & $(-)$ & $\begin{array}{l}\text { SE, Pneumo- } \\
\text { cephalus }\end{array}$ & Craniotomy & Dead \\
\hline
\end{tabular}


Recent experimental hematogenous meningitis models have indicated that the primary site of entry of circulating $E$. coli into the CNS is the cerebral vasculature, not the choroid plexus [3]. Nevertheless, hematogenous brain abscess formation owing to $E$. coli infection is rare. Bakker et al. [4] reported that autopsy of a patient with $E$. coli-induced subdural empyema showed no obvious inflammation in the brain parenchyma. In our reviewed cases, seven of nine patients showed subdural empyema. These findings suggest the presence of key mechanisms preventing $E$. coli infection in the brain parenchyma. In an in vitro blood-brain barrier model using human brain microvascular endothelial cells (HBMECs), E. coli was shown to invade and internalize the HBMECs as membrane-bound vacuoles with no changes in the integrity of the HBMEC monolayer [3]. Moreover, E. coli enters the CNS with no changes in the blood-brain barrier permeability and no concomitant presence of host inflammatory cells [4]. Once $E$. coli invades the brain parenchyma, microglia, the resident macrophage population in the CNS, may play a key role in recognizing and eliminating the microbes via Toll-like receptors or phagocytic receptors [13]. Additionally, activated microglia produce various pro-inflammatory cytokines, leading to the activation and chemotaxis of peripheral immune cells; however, their phagocytic or killing activity toward microbes is less potent than that of polymorphonuclear leukocytes [13]. A recent study showed that microglia and astrocytes are specifically activated soon after bacterial invasion into the CNS parenchyma [13]. The above findings indicate that impaired glial cell function or an impaired immune response induced by glial cells may contribute to $E$. coli infection in the CNS parenchyma.

Notably, a preceding $E$. coli infection of a simple right renal cyst might have caused the bacteremia and subsequent brain abscess in the present case. Simple renal cysts are usually observed as unilateral and solitary lesions, and the prevalence rate ranges from 7 to $10 \%$, increasing with age [14]. Major complications of simple renal cysts, such as hemorrhage, infection, and rupture, are rare events seen in only $2-4 \%$ of affected patients [14]. Suwabe et al. [15] showed that renal cysts with high intensity, a fluid-fluid level, or wall thickening on diffusion-weighted imaging suggest the presence of a cyst infection in patients with autosomal dominant polycystic kidney disease. A heterogeneous internal cyst density with no enhancement on $\mathrm{CT}$ also suggests a cyst infection [15]. Symptoms of cyst infection are nonspecific. Especially in patients with autosomal-dominant polycystic kidney disease, the most conspicuous symptom is fever; in general, abdominal pain and frank hematuria are not observed [15]. Cyst puncture and aspiration can be diagnostic and may circumvent the need for surgical procedures such as nephrectomy [14]. Physicians should know that cyst infections may cause serious complications, even in patients with simple renal cysts.

\section{Conclusion}

We experienced a rare case of a brain abscess following a simple E. coli renal cyst infection. Brain abscess formation owing to hematogenous spread of $E$. coli is very rare. Simple renal cyst infection is also rare, but can cause serious complications. Immunosuppression may contribute to the onset of intracranial E. coli abscesses, such as brain abscesses and subdural empyema. Advanced age may also be a potent risk factor.

\section{Consent}

Written informed consent was obtained from the patient for publication of this case report and any accompanying images. A copy of the written consent is available for review by the Editor of this journal.

\section{Abbreviations}

CNS: Central nervous system; CRP: C-reactive protein; CSF: Cerebrospinal fluid; CT: Computed tomography; E. coli: Escherichia coli; GCS: Glasgow coma scale; HBMEC: Human brain microvascular endothelial cell; MRI: Magnetic resonance imaging; WBC: White blood cell.

\section{Competing interests}

The authors declare that they have no competing interests.

\section{Authors' contributions}

NA drafted the manuscript. TO, MT, and TH collected the patient data and monitored the patient throughout the whole follow-up period. $\mathrm{KI}$ and $\mathrm{YK}$ edited the manuscript. MK participated in the study design and coordination and helped to draft the manuscript. All authors have read and approved the final manuscript.

\section{Acknowledgements}

The authors would like to thank Dr. Jun Aoki for his assistance in preparing the radiographic images and Miss Hisae Kuribara for her secretarial assistance under the financial support provided by Social Insurance Gunma Chuo General Hospital.

\section{Author details}

'Departments of Internal Medicine, 1-7-13 Koun-cho, Maebashi, Gunma 371-0025, Japan. Neurology, Gunma Chuo Hospital, 1-7-13 Koun-cho, Maebashi, Gunma 371-0025, Japan. ${ }^{3}$ Department of Medicine and Biological Science, Graduate School of Medicine, Gunma University, 3-39-22

Showa-machi, Maebashi, Gunma 371-8511, Japan.

Received: 24 February 2014 Accepted: 12 June 2014

Published: 16 June 2014

\section{References}

1. Alvis Miranda H, Castellar-Leones SM, Elzain MA, Moscote-Salazar LR: Brain abscess: current management. J Neurosci Rural Pract 2013, 4(Suppl 1):S67-S81.

2. Helweg-Larsen J, Astradsson A, Richhall H, Erdal J, Laursen A, Brennum J: Pyogenic brain abscess, a 15 year survey. BMC Infect Dis 2012, 12:332.

3. Kim KS: Current concepts on the pathogenesis of Escherichia coli meningitis: implications for therapy and prevention. Curr Opin Infect Dis 2012, 25:273-278.

4. Bakker S, Kluytmans J, den Hollander JC, Lie ST: Subdural empyema caused by Escherichia coli: hematogenous dissemination to a preexisting chronic subdural hematoma. Clin Infect Dis 1995, 21:458-459. 
5. Hirano A, Takamura T, Murayama N, Ohyama K, Matsumura S, Niwa J: Subdural abscess following chronic subdural hematoma. No Shinkei Geka 1995, 23:643-646 (in Japanese).

6. Rickert CH, August C, Brandt M, Wagner V, Paulus W: Cerebral malakoplakia associated with Escherichia coli infection. Acta Neuropathol 2000, 99:595-598.

7. Nishi H, Shibagaki Y, Hatakeyama S, Ito T, Nagata T, Ohno M, Tojo A, Nangaku M, Fujita T: Metastatic intracranial subdural empyema from renal cyst infection in autosomal dominant polycystic kidney disease. Nephrol Dial Transplant 2005, 20:2820-2823.

8. Bachmeyer C, Logak M, Ammouri W, Blanc AS: Spontaneous Escherichia coli meningitis with subdural empyema in an adult. South Med J 2005, 98:1225-1226.

9. Doepp F, Schreiber SJ, Wandinger KP, Trendelenburg G, Valdueza JM: Multiple brain abscesses following surgical treatment of a perianal abscess. Clin Neurol Nerosurg 2006, 108:187-190.

10. Adamides AA, Goldschlager T, Tulloch SJ, McMahon JH: Pneumocephalus from gas-forming Escherichia coli subdural empyema. Br J Neurosurg 2007, 21:299-300.

11. Narita E, Maruya J, Mishimaki K, Heianna J, Miyauchi T, Nakahata J, Kitahara $H$, Minakawa T: A case of infected subdural hematoma diagnosed by diffusion-weighted imaging. Brain Nerve 2009, 61:319-323 (in Japanese).

12. Redhu R, Shah A, Jadhav M, Goel A: Spontaneous tension pneumocephalus in a patient with subdural empyema. J Clin Neurosci 2011, 18:1123-1124.

13. Mariani MM, Kielian T: Microglia in infectious diseases of the central nervous system. J Neuroimmune Pharmacol 2009, 4:448-461.

14. Eknoyan G: A clinical view of simple and complex renal cysts. J Am Soc Nephrol 2009, 20:1874-1876.

15. Suwabe T, Ubara Y, Sumida K, Hayami N, Hiramatsu R, Yamanouchi M, Hasegawa E, Hoshino J, Sawa N, Saitoh S, Okuda I, Takaichi K: Clinical features of cyst infection and hemorrhage in ADPKD: new diagnostic criteria. Clin Exp Nephrol 2012, 16:892-902.

doi:10.1186/1471-2377-14-130

Cite this article as: Akuzawa et al:: Secondary brain abscess following simple renal cyst infection: a case report. BMC Neurology 2014 14:130.

\section{Submit your next manuscript to BioMed Central and take full advantage of:}

- Convenient online submission

- Thorough peer review

- No space constraints or color figure charges

- Immediate publication on acceptance

- Inclusion in PubMed, CAS, Scopus and Google Scholar

- Research which is freely available for redistribution 